\title{
JOURNAL

\section{Lumbar spinal muscle activation synergies predicted by multi-criteria cost function}

\author{
Ian A.F. Stokes*, Mack Gardner-Morse \\ Department of Orthopaedics and Rehabilitation, University of Vermont, Burlington, VT 05405-0084, USA \\ Accepted 13 February 2001
}

\begin{abstract}
The hypothesis that control of lumbar spinal muscle synergies is biomechanically optimized was studied by comparing EMG data with an analytical model with a multi-component cost function that could include (1) trunk displacements, (2) intervertebral displacements, (3) intervertebral forces; (4) sum of cubed muscle stresses, and (5) eigenvalues for the first two spinal buckling modes. The model's independent variables were 180 muscle forces. The 36 displacements of 6 vertebrae were calculated from muscle forces and the spinal stiffness. Calculated muscle activation was compared with EMG data from 14 healthy human subjects who performed isometric voluntary ramped maximum efforts at angles of $0^{\circ}, 45^{\circ}, 90^{\circ}, 135^{\circ}$ and $180^{\circ}$ to the right from the anterior direction. Muscle activation at each angle was quantified as the linear regression slope of the RMS EMG versus external force relationship, normalized by the maximum observed EMG.

There was good agreement between the analytical model and EMG data for the dorsal muscles when the model included either minimization of intervertebral displacements or minimization of intervertebral forces in its cost function, but the model did not predict a realistic level of abdominal muscles activation. Agreement with EMG data was improved with the sum of the cubed muscle stresses added to the cost function. Addition of a cost function component to maximize the trunk stability produced higher levels of antagonistic muscle activation at low efforts than at greater efforts. It was concluded that the muscle activation strategy efficiently limits intervertebral forces and displacements, and that costs of higher muscle stresses are taken into account, but stability does not appear to be maximized. Trunk muscles are apparently not controlled solely to optimize any one of the biomechanical costs considered here. (C) 2001 Elsevier Science Ltd. All rights reserved.
\end{abstract}

Keywords: Muscles; Human trunk; Lumbar spine; Neuromuscular control; Optimization

\section{Introduction}

The number of muscles crossing the lumbar spine exceeds 180 (Stokes and Gardner-Morse, 1999) and this is much greater than the number of degrees of freedom (6 per vertebra) in a static analysis of the lumbar spine. This means that there is a highly redundant number of muscle activation patterns that could be used to satisfy equilibrium. In indeterminant biomechanical analyses, it is usually assumed that the CNS control of trunk muscle activation strategy is in some way optimal, compatible with equilibrium and other constraints (Dul et al., 1984). In optimization models, several different physiological cost functions have been proposed, including to mini-

\footnotetext{
*Corresponding author. Tel.: +1-802-656-2250; fax: + 1-802-6564247.

E-mail address: stokes@med.uvm.edu (I.A.F. Stokes)
}

mize intervertebral forces, minimize muscle forces (Bean et al., 1988; Crowninshield and Brand, 1981; Hughes et al., 1994; Schultz, 1990), minimize the total of muscle and joint forces (Yettram and Jackman, 1982) or optimize spinal displacements (Skogland and Miller, 1980; Wynarsky and Schultz, 1991). If more than one variable is entered into the cost function, then weighting factors must be assigned to each component. Alternatively, the components of the optimization can be analyzed sequentially (Bean et al., 1988).

Although optimization models of the spine have considered the physiological costs of activating muscles and loading the skeleton, these physiological costs have generally been considered singly or as competing objectives, while in reality several costs may be taken into account in establishing an efficient muscle activation strategy. Furthermore, the stability of the spinal column has not previously been entered into optimization 
models as an objective. The spine is inherently unstable (Crisco et al., 1992), and a combination of muscle forces, muscle stiffness (Bergmark, 1989; Cholewicki and McGill, 1996; Crisco et al., 1992; Gardner-Morse et al., 1995), and motion segment stiffness (Panjabi, 1992) is required to prevent buckling instability. Stability analyses have predicted that antagonistic muscle coactivation would increase stability (GardnerMorse and Stokes, 1998), and the existence of such antagonistic activity has been confirmed in electromyographical studies of muscle activation (Lavender et al., 1992; Thelen et al., 1995). The fact that most optimization models do not predict antagonistic muscle coactivation has been seen as a limitation of these models.

This paper tests the hypothesis that muscle activation around the lumbar spine is biomechanically optimized to minimize intervertebral or muscle forces, to minimize local or global spinal displacements, to maximize spinal stability, or to optimize combinations of these. This was done by comparing EMG data against predictions of muscle force distributions obtained analytically by minimizing a cost function that was the weighted sum of several biomechanical variables in an anatomically complex biomechanical model.

\section{Methods}

Analyses were performed with a lumbar spine model having 36 degrees of freedom (a rigid lumped thorax and 5 lumbar vertebrae each having 6 degrees of freedom relative to the fixed sacrum/pelvis). The intervertebral joints had stiffness represented by beam elements (Stokes and Gardner-Morse, 1995). There were 180 muscles whose attachment points and physiologic crosssectional areas were taken from Stokes and GardnerMorse (1999). The 180 muscle forces were the independent unknowns in the optimization that were calculated by minimizing the cost function. Vertebral displacements and intervertebral motions were additional dependent variables in the model that were calculated from the stiffness matrix of the model and the externally applied and muscular forces.

The cost function was formulated as the weighted sum of five components (Eq. (1)). The five components were: the sum of squared global displacements, sum of squared local intervertebral displacements, sum of squared intervertebral forces, sum of muscle stress cubed and the sum of the exponential of the negated values of the smallest two eigenvalues of buckling modes of the trunk. The smallest eigenvalue must be positive $(>0)$ for stability (Bergmark, 1989). These five components of the objective function correspond to minimizing changes of trunk posture, minimizing relative displacements between vertebrae, minimizing intervertebral forces and moments, avoiding high muscle forces (and hence muscle fatigue) and maximizing the smallest eigenvalues (and hence spinal stability).

Thus the problem is formulated as:

Minimize $F=\sum_{i=1}^{5} W_{i} f_{i}$

using

$$
\begin{gathered}
f_{1}=\sum_{j=1}^{36} w_{1_{j}} \Delta_{j}^{2}, f_{2}=\sum_{j=1}^{36} w_{2_{j}} \delta_{j}^{2}, f_{3}=\sum_{j=1}^{72} w_{3_{j}} f_{s_{j}}^{2}, \\
f_{4}=\sum_{j=1}^{180} \sigma_{m_{j}}^{3}, f_{5}=\mathrm{e}^{-\lambda_{1}}+\mathrm{e}^{-\lambda_{2}}
\end{gathered}
$$

where $W_{i}$ are the relative weights of each of the five components of the cost function, $w_{1}$ are the relative weights of the individual global displacements and rotations of the trunk and lumbar vertebrae $\Delta, w_{2}$ are the relative weights of the individual intervertebral displacements and rotations $\delta, w_{3}$ are the relative weights of the individual intervertebral forces and moments $f_{s}, \sigma_{m}$ are the muscle stresses, $\lambda_{1}$ is the smallest eigenvalue and $\lambda_{2}$ is the next smallest eigenvalue of the Hessian matrix of partial second derivatives of the trunk and lumbar spine potential energy with respect to each degree of freedom (Bergmark, 1989).

In both the global and local displacement components of the cost function, the subweights $w_{1_{j}}$ and $w_{2_{j}}$ were such that $1 \mathrm{~mm}$ of displacement and $1^{\circ}$ of rotation were weighted equally. In the global displacements, only the displacements of the lumped thorax were considered. The relative subweights of the intervertebral forces and moments $w_{3_{j}}$ were such that $3 \mathrm{~N}$ of force and $1 \mathrm{Nmm}$ of moment were weighted equally. These values were based on the presumed safe limits of intervertebral loads being approximately $3000 \mathrm{~N}$ for forces and $9000 \mathrm{~N} \mathrm{~mm}$ for moments (ratio of 1 to 3 ). The displacements and intervertebral forces were squared so that positive and negative values would contribute equally in the cost function.

The trunk stability was measured by identifying eigenvalues of buckling modes of the trunk from the Hessian matrix of partial second derivatives of the trunk and spine potential energy with respect to each degree of freedom (Bergmark, 1989). The trunk potential energy is stored in the elastic intervertebral joints of the spine and in displacements of the activated muscles. For these analyses, the muscle stiffness $k$ was considered to increase with muscle force $(F)$ using the relationship $k=$ $q F / l$ (Bergmark, 1989) where $l$ is the resting length of the muscle and $q$ is a dimensionless constant. Here $q$ was assigned a value of 5.0 based on the range of experimentally determined values reported by Crisco and Panjabi (1991). To improve the numerical stability and convergence of the models that included stability in the cost function, the two smallest eigenvalues $\lambda_{1}$ and $\lambda_{2}$ were used in calculating $f_{5}$. The exponential function of the negated smallest eigenvalues penalizes negative 
values, and favours a large positive value of the smallest eigenvalue (i.e. a stable model).

The bounds for permissible muscle forces were greater than or equal to zero and less than a maximum that was equal to the muscle physiological cross-sectional area (PCSA) multiplied by the assumed maximum muscle stress (460 kPa (Gardner-Morse et al., 1995)).

To simulate the experiments with human subjects (see below), solutions were calculated for external loading at $\mathrm{T} 12$ by a horizontal force of 0 to $300 \mathrm{~N}$ in steps of $50 \mathrm{~N}$ acting at angles of $0^{\circ}, 45^{\circ}, 90^{\circ}, 135^{\circ}$ or $180^{\circ}$ degrees to the anterior direction, on the right side. Initial loading (the weight of the trunk and any preactivation of the muscles) was omitted from the analyses.

To investigate the contribution of each component of the cost function, runs were made with the weights $W_{i}$ in Eq. (1) set sequentially for each component to 1 , with the other four weights set to zero. The solutions for these single component cost functions were examined for agreement with the experimental findings (see below). Then combinations of two or three cost function components were investigated, using the same criteria to evaluate the realism of the predicted muscle forces and spinal displacements. The relative weights of the two cost function subcomponents were set so that each contributed approximately equally to the total cost over the series of simulations at different angles and effort magnitudes.

The simulations were performed using the MINOS optimization program (Operation Research Laboratory, Stanford University, Stanford, CA). This solution method used the sensitivity of the cost function to the muscle forces variables. These gradients with respect to each muscle force were calculated analytically, except for the stability objective where an approximate gradient was calculated based on perturbation methods (Inman, 1989).

For the experimental determination of comparable muscle activation patterns, 14 subjects $(8$ male, 6 female; ages 20-26 years; bodymass $75 \pm 14 \mathrm{~kg}$ ) were studied with IRB approval and informed consent. Each stood in an apparatus that had pads pressing on the ASIS and sacrum to minimize motion of the pelvis. They performed ramped isometric efforts up to a voluntary maximum. The resistance was provided by a harness around the thorax connected to a cable that was attached to each of a series of five anchor points on the wall, to vary the angle of pull in $45^{\circ}$ increments from $0^{\circ}$ to $180^{\circ}$ to the anterior direction. A load cell in the cable recorded the force generated. EMG electrodes recorded signals from six right and left pairs of muscles (rectus abdominis, internal and external obliques, longissimus, iliocostalis and multifidus at the level of L4). Electrode types and positioning were as reported in Stokes et al. (2000). Surface electrodes were used, except for multifidus, where fine wire electrodes were employed.
EMG and load cell signals were recorded digitally at $1024 \mathrm{~Hz}$ (first 7 subjects) or $2048 \mathrm{~Hz}$ (subsequent subjects). The EMG signals were then converted to RMS signals with a $293 \mathrm{~ms}$ moving average boxcar filter.

The activation of each muscle at each angle was expressed as the slope of the RMS EMG-force linear regression relationship. These muscle activation slopes were normalized by dividing by the maximum recorded EMG value for that muscle in all the experiments. For comparison, the muscle activations from the model were measured by the linear regression slopes of activation versus force generated, and normalized by the muscle's maximum force. Since the model included 180 muscles and only 12 muscles were recorded via EMG, the modelpredicted activity levels were averaged within each anatomical group, after weighting the activity of each muscle component by its PCSA for comparisons with experimental data.

Each analytical solution was compared with experimental findings, using the following criteria for agreement:

1. The muscle forces increased monotonically with effort in each loading direction.

2. The model was structurally stable (eigenvalues $>0$ ).

3. Spinal displacements were in a plausible range. Intervertebral rotations greater than 5 degrees and shear displacements greater than $4 \mathrm{~mm}$ were considered implausible, because they are likely to cause soft tissue injury.

4. Numbers of active muscles.

5. Good quantitative fit between muscle activation in the model and that found experimentally. For this comparison the sum of squared weighted activationforce slope differences (SSSD) was used in the following measure of fit:

$\mathrm{SSSD}=\sum_{i=1}^{60}\left(s_{\mathrm{e}_{i}}-s_{\mathrm{a}_{i}}\right)^{2} * S_{\mathrm{e}_{i}}$

where for each muscle, $s_{\mathrm{a}}=$ analytical activationforce slope; $s_{\mathrm{e}}=$ experimental activation-force slope.

The summation was over 12 muscles at each of 5 angles (60 values). Each squared slope difference was weighted by the magnitude of the experimental activation-force slope $s_{\mathrm{e}}$. The units were $\mathrm{kN}^{-3}$.

The set of component cost functions that most likely are used in the hypothesized optimization strategy were identified by first finding the single components of the cost function that gave reasonable solutions for the muscle forces and displacements. Then additional components were added to identify the combinations of two cost function components that improved model agreement with experimental muscle activation data. 


\section{Results}

The experimental muscle activation slopes for all the muscles (except multifidus at $180^{\circ}$ ) had positive activation slopes (Fig. 1). This indicates that those muscles, even at angles where they are antagonistic, increased their level of activation with increasing effort. The activation for the internal and external oblique muscles was least variable with the angle, indicating that these muscles were coactivated almost independent of the direction of the generated force.

1. Minimize global displacement: Including only the global displacements of the thorax in the cost function did not produce good agreement with experimental data. It produced generally non-linear increases in muscle activation with external efforts, and several negative slopes in these relationships. The SSSD was 16.5 (Table 1), indicating a relatively close agreement
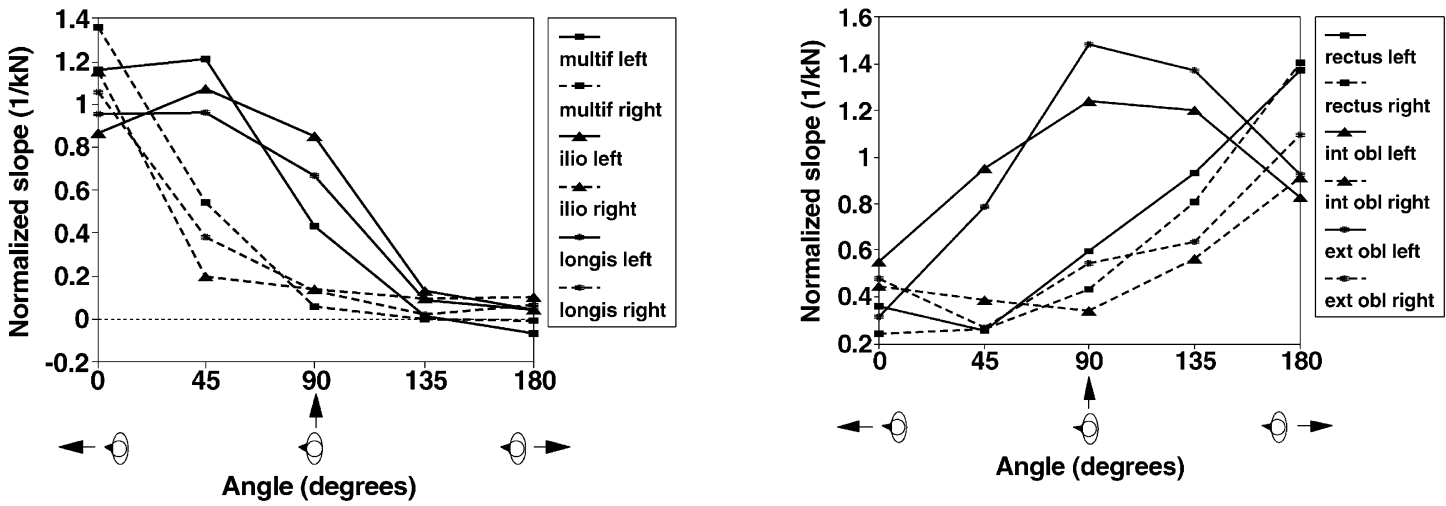

Fig. 1. Experimentally measured muscle activation expressed as the slope of the RMS EMG versus force relationships as a function of the angle of the cable. Each slope was derived from the regression relationship found between RMS EMG and force generated by the subject. The values plotted are averaged from values for 14 subjects and 3 trials at each angle. The units are proportion of maximum EMG/kN. (Left) extensor muscles and (right) abdominal muscles.

Table 1

Summary of observed findings from nine different optimization models with one or two cost function components

\begin{tabular}{|c|c|c|c|c|c|c|c|c|c|}
\hline & \multicolumn{3}{|l|}{$\begin{array}{l}\text { Cost function } \\
\text { includes global } \\
\text { displacements }\left(f_{1}\right)\end{array}$} & \multicolumn{3}{|c|}{$\begin{array}{l}\text { Cost function } \\
\text { includes intervertebral } \\
\text { displacements }\left(f_{2}\right)\end{array}$} & \multicolumn{3}{|c|}{$\begin{array}{l}\text { Cost function } \\
\text { includes intervertebral } \\
\text { forces }\left(f_{3}\right)\end{array}$} \\
\hline & Alone & $\begin{array}{l}\text { With } \\
\text { muscle } \\
\text { stress }^{3}\left(f_{4}\right)\end{array}$ & $\begin{array}{l}\text { With } \\
\text { stability } \\
\left(f_{5}\right)\end{array}$ & Alone & $\begin{array}{l}\text { With } \\
\text { muscle } \\
\text { stress }^{3}\left(f_{4}\right)\end{array}$ & $\begin{array}{l}\text { With } \\
\text { stability } \\
\left(f_{5}\right)\end{array}$ & Alone & $\begin{array}{l}\text { With } \\
\text { muscle } \\
\text { stress }^{3}\left(f_{4}\right)\end{array}$ & $\begin{array}{l}\text { With } \\
\text { stability } \\
\left(f_{5}\right)\end{array}$ \\
\hline SSSD value & 16.5 & 15.5 & 22.0 & 18.0 & 14.7 & 20.7 & 16.4 & 14.9 & 21.1 \\
\hline $\begin{array}{l}\text { Linear relationship } \\
\text { of muscle activation } \\
\text { to effort? }\end{array}$ & No & Improved $^{\mathrm{a}}$ & No & Yes & Better $^{\mathrm{a}}$ & Worse $^{\mathrm{a}}$ & Yes & Better $^{\mathrm{a}}$ & Similar $^{\mathrm{a}}$ \\
\hline $\begin{array}{l}\text { Abdominal } \\
\text { muscles active? }\end{array}$ & $\begin{array}{l}\text { Only external } \\
\text { obliques }\end{array}$ & Similar $^{\mathrm{a}}$ & Similar $^{\mathrm{a}}$ & $\begin{array}{l}\text { External + some } \\
\text { internal obliques. } \\
\text { (no rectus) }\end{array}$ & Similar ${ }^{\mathrm{a}}$ & Similar $^{\mathrm{a}}$ & $\begin{array}{l}\text { External + some } \\
\text { internal obliques. } \\
\text { (no rectus) }\end{array}$ & Similar $^{\mathrm{a}}$ & Similar $^{\mathrm{a}}$ \\
\hline $\begin{array}{l}\text { Negative rate of } \\
\text { muscle activation } \\
\text { with effort? }\end{array}$ & Several & None & Several & None & None & $\begin{array}{l}\text { Iliocostalis, } \\
\text { internal } \\
\text { and external } \\
\text { obliques }\end{array}$ & Some & None & Several \\
\hline $\begin{array}{l}\text { Displacements } \\
\text { plausible? }\end{array}$ & $\begin{array}{l}\text { Yes, except } 5.4^{\circ} \\
\text { flexion at } \mathrm{L} 2-3 \text {; } \\
4.6 \mathrm{~mm} \text { shear } \\
\text { at } \mathrm{L} 3-4\end{array}$ & Yes & Yes & Yes & Yes & Yes & Yes & Yes & Yes \\
\hline
\end{tabular}

${ }^{\text {a }}$ Compared with results from the corresponding single cost function model. 
between predicted and experimental muscle activation. However, some intervertebral displacements were greater than the physiological range. There was $5.4^{\circ}$ of flexion rotation at L2-3 (for an external force of $200 \mathrm{~N}$ at $0^{\circ}$ ) and $4.6 \mathrm{~mm}$ of anterior shear displacement at L3-4 (for a force of $300 \mathrm{~N}$ at $0^{\circ}$ ). Therefore this solution was considered to be a poor match with experimental observations and we rejected the hypothesis that global displacements alone are minimized in the lumbar spinal muscular activation strategy.

2. Minimize local (intervertebral) displacements: Including only the local (intervertebral) displacements in the cost function produced solutions with near-linear relationships between muscle activation and effort and no negative activation-force slope values. However, this cost function never predicted activation of rectus abdominis, right psoas or right quadratus lumborum. There were no excessive trunk or intervertebral displacements. However, the SSSD was 18.0 (Table 1), indicating a relatively poor agreement between predicted and experimental muscle activation. Therefore we also rejected the hypothesis that the muscle activation strategy is based only on minimizing intervertebral displacements.

3. Minimize local (intervertebral) forces: Including only the local (intervertebral) forces in the cost function produced solutions that were similar to those with local displacements in the cost function; this was expected since these variables were related to each other by the spinal stiffness matrix. Again, there were no excessive trunk or intervertebral displacements. The SSSD was 16.4 (Table 1). Therefore, these simulations were considered plausible, and with reasonable agreement with experimental data, so it is possible that the muscle activation strategy includes minimization of intervertebral forces.

4. Minimize muscle stresses cubed: Including only muscle stresses in the cost function resulted in large unrealistic joint displacements (and hence large intervertebral elastic forces). We therefore reject the hypothesis that muscle stresses alone are minimized in the muscle activation strategy.

5. Maximize stability: Weighting only stability generated implausibly large intervertebral displacements especially at low efforts, and there was an unrealistically large amount of muscle coactivation with up to $20 \%$ activation of the oblique muscles. Maximization of trunk stability alone as a muscle activation strategy is also rejected.

Because the three subobjectives $f_{1}, f_{2}$, and $f_{3}$, gave the most realistic solutions when included alone in the cost function, these cost function components were then used in conjunction with each of the other two, to identify pairs that gave better agreement than single cost function components alone. When the muscle stress subcomponent $f_{4}$ was added to the global displacement $\left(f_{1}\right)$, the local displacement subcomponent $\left(f_{2}\right)$ or the local forces $\left(f_{3}\right)$ subcomponents, the combinations gave improved agreement with experimental results. The opposite trend was observed for the stability subcomponent $\left(f_{5}\right)$. Overall, the best agreement with the experimental data was obtained with the muscle stress cubed subobjective $\left(f_{4}\right)$ combined with the intervertebral displacement subobjective $\left(f_{2}\right)$ (Figs. 2 and 3 ).

Adding the stability subobjective $\left(f_{5}\right)$ to any of the first 3 subobjectives $f_{1}, f_{2}$, or $f_{3}$ predicted greater muscle activation, including antagonism, but this occurred preferentially at low efforts. This predicted pattern of muscle activations was unrealistic because many muscles were observed in the model to have decreased activation with increasing effort, i.e. the number of negative slopes $\mathrm{s}_{\mathrm{a}}$ increased. The results of these simulations with pairs of subcomponents entered into the cost function are summarized in Table 1.

In all of the simulations with different cost-function formulations there was consistently no activity in rectus abdominis, although this muscle was forced to be active in an exploratory simulation with the area of the obliques artificially reduced by a factor of 10 . In all simulations the trunk was stable (i.e. eigenvalues $>0$ ). For the two plausible single cost function component simulations in Table 1 there were between 32 and 64 active muscles for a $300 \mathrm{~N}$ horizontal force external loading. The greatest number of activated muscles was observed at $0^{\circ}$ external force direction, with fewer numbers at other angles. Most of the muscles that were activated in these simulations were also maximally active (between 27 and 54 maximally active muscles for $300 \mathrm{~N}$ force). The inclusion of muscle stresses in the cost function increased the number of active muscles to the range between 35 and 90 at $300 \mathrm{~N}$ but the number of maximally active muscles reduced to the range between 7 and 20. The inclusion of stability in the cost function only slightly increased the number of active muscles. Thus only between $1 / 3$ and $1 / 2$ of the 180 muscles were activated at any given angle in these simulations.

\section{Discussion}

The simulations with single component cost functions did not agree with experimental data as well as the simulations made with a combination of the muscle stresses and either intervertebral forces or intervertebral displacements minimized. The multi-component cost function analyses simulated a muscle activation strategy in which several conditions must be respected, notably that the spine must be in equilibrium, must be stable, that muscle stresses must be between zero and an upper physiological limit, and that joint forces and displacements must be limited. Plausible model predictions were only obtained from the models having a single cost 

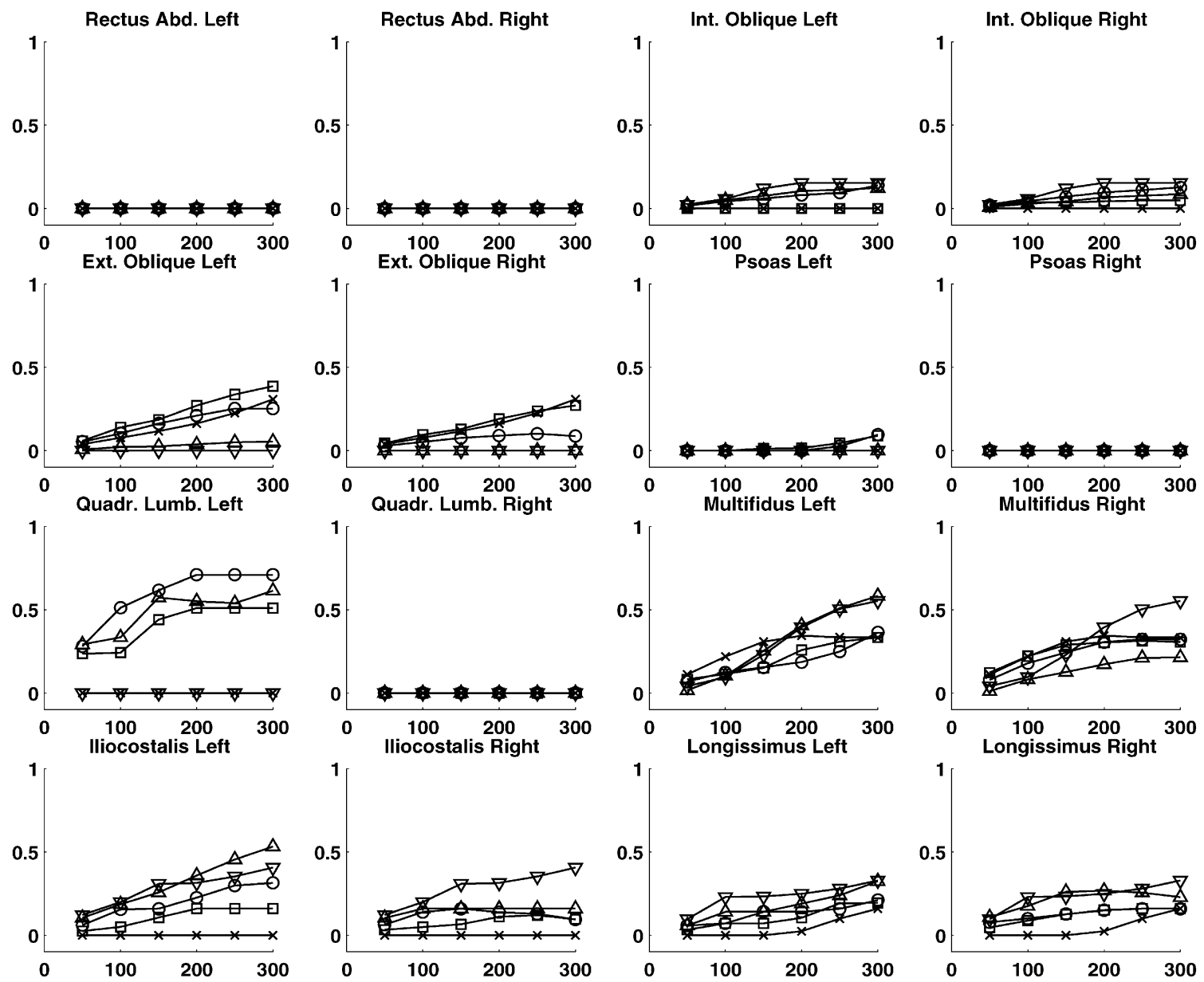

Fig. 2. Muscle activation (proportion of maximum-vertical axes) as a function of force magnitude in Newtons (horizontal axes) predicted by the model with the intervertebral displacements and muscle stress cubed subobjectives included in the cost function. $\left(\nabla=0^{\circ}\left(\right.\right.$ extension); $\Delta=45^{\circ} ; \bigcirc=90^{\circ}$ (lateral); $\square=135^{\circ} ; \times=180^{\circ}$ (flexion)).

function component when that component was the intervertebral displacements or the intervertebral forces. Because the intervertebral forces and displacements were related to each other by the spinal stiffness matrix that includes 'off-diagonal' coupled terms, these two strategies gave similar though not identical results.

In disagreement with previous reports (Crowninshield and Brand, 1981; Hughes et al., 1994; van Dieën, 1997), realistic predictions of muscle forces were not obtained by minimizing muscle stresses alone. Our simulations with only muscle stress cubed in the cost function predicted very large intervertebral rotations and consequently large intervertebral torques, instead of torques generated by muscular forces. This muscle stress cost function has been reported previously (Hughes et al., 1994; van Dieën, 1997) as giving realistic muscle force predictions in the spine, but in models that do not consider intervertebral flexibility and the associated forces and displacements generated by elastic deformation of the spinal column, and with only three degrees of freedom (rotations of a single joint) that were assumed to be of zero magnitude. Therefore the present model is considered to be more realistic, and requires intervertebral displacements or forces in its cost function, along with the muscle stresses.

It was expected that introducing the trunk stability into the cost function would improve the model by forcing antagonistic muscle activation. For example, antagonistic activation of abdominal muscles is thought to increase trunk stability. Stability has not been investigated previously as a variable to optimize trunk muscle function. However, including stability with other cost function components decreased the realism of the models, with abdominal muscle co-activation occurring preferentially when the external effort was small (negative activation slopes). It may be that in reality stability is not maximized, but rather is set to a target or minimum level. The absence of rectus abdominis activation in the model may be a result of model anatomical simplifications (absence of abdominal muscle curvature, and simplifications of the anterior attachments of the oblique abdominal muscles). 
Rectus Abd.
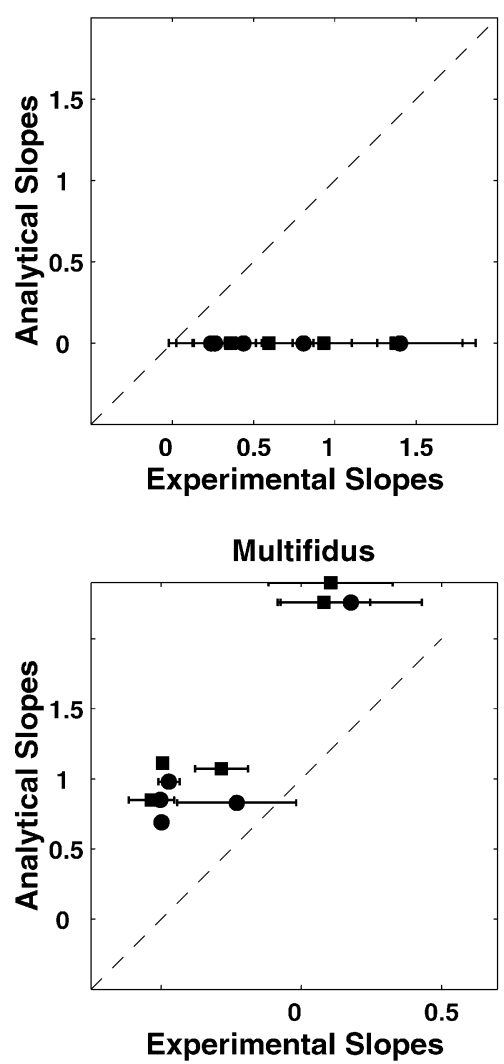

Int. Oblique

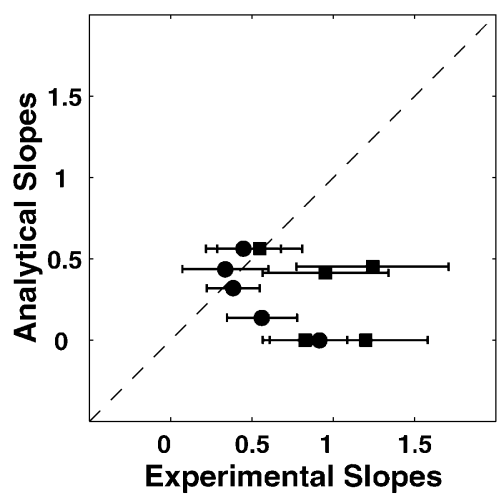

Iliocostalis

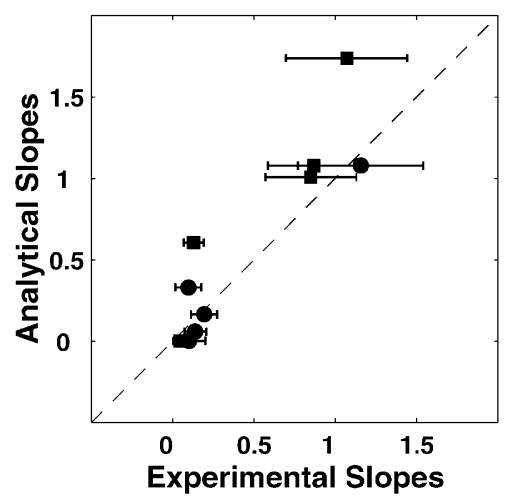

Ext. Oblique

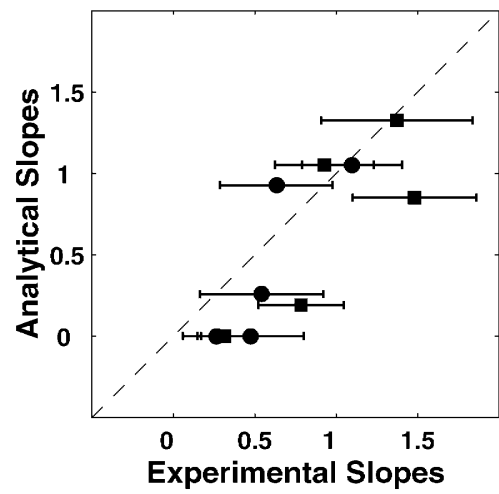

Longissimus

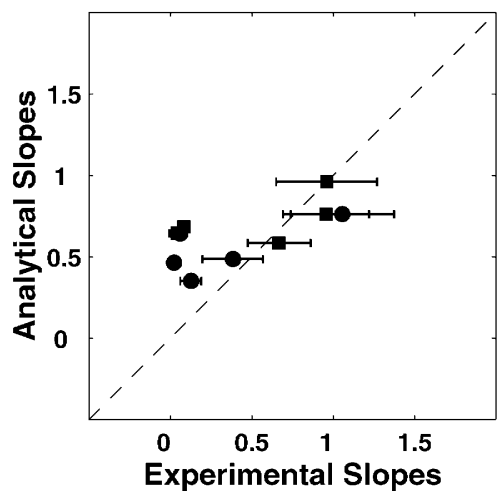

Fig. 3. Muscle activations expressed as the slopes of activation-force regression relations. Each point corresponds to a single direction (angle) of the external force. Horizontal axis: experimental slopes; vertical axis: slopes predicted analytically. The broken line indicates the line of perfect agreement. The error bars are the $95 \%$ confidence interval for the mean experimental values. The analytical results are those predicted by the model with the intervertebral displacements and muscle stress cubed subobjectives included in the cost function. $(\mathbf{O}=$ right muscles; $\square=$ left muscles).

Although this study aimed to find biomechanical parameters that are optimized in the trunk, the analyses were limited to the hypothetical functions of a limited number of biomechanical variables identified in Eq. (1). This kind of study (comparison of experimental data with a predictive model) can generate evidence to refute a hypothesis. Although a fit between a model strategy and experiments does not necessarily imply that the modelled strategy is correct, the hypothetical strategies that predict implausible results in a model do serve to disprove those hypotheses subject to the simplifications of the model.

Only quasi-static isometric conditions were studied here in which muscles were at their resting lengths. The experimental studies similarly were isometric; it may be that muscle redundancy is exploited differently in other circumstances that we did not investigate. Also, it was difficult to evaluate the models with the practical limitation of 12 channels of EMG. The criteria for evaluating good agreement between models and experimental data required that we averaged the activation within groups of muscles and also that we summarized model performance over a broad range of external force magnitudes and directions to obtain a scalar measure of agreement. Because the relationships between RMS EMG and the external force were observed to be nearly linear during the ramped voluntary increase of force, a linear, monotonic increase of muscle activation with effort was considered to indicate a realistic performance of the model. The linear gradients of the muscle activation versus generated effort relationships were used to summarize the muscle's activation.

If the CNS were continuously evaluating cost functions to control muscles optimally, then a more complex nonlinear activation-external force relationship might be generated, but this seems unlikely because of the computational effort required. For example, models based on minimizing spinal compression force predict that as the amount of effort increases in any particular task or loading direction, advantageously placed muscles are activated first, and are augmented by other muscles for larger efforts, thus muscle activation increases discontinuously with effort. This is contradicted by the experimental recordings of EMG versus effort, and those of comparable studies (Lavender et al., 1992; Thelen et al., 1995) that report small changes in 
the relative activations of trunk muscles with increasing effort. Instead, the idea of a preset pattern of synergies that remains constant with changing effort is attractive, but this would not always produce biomechanically optimal strategies.

The analyses reported here give insights into the way in which the redundant number of muscles of the trunk is controlled in quasi-static situations. Here the experimentally observed muscle activation patterns are close to those predicted analytically by minimizing intervertebral forces or displacements, together with minimized muscle stresses. Therefore these biomechanical parameters are probably close to optimal values in isometric efforts. We cannot be certain whether the CNS set these parameters as goals to be optimized, or arrived at them fortuitously.

\section{Acknowledgements}

Supported by NIH R01 AR 44119. Jeffrey P. Laible assisted in the model development. David F. Norton performed the human subject experiments.

\section{References}

Bean, J.C., Chaffin, D.B., Schultz, A.B., 1988. Biomechanical model calculation of muscle contraction forces: a double linear programming method. Journal of Biomechanics 21 (1), 59-66.

Bergmark, A., 1989. Stability of the lumbar spine. A study in mechanical engineering. Acta Orthopaedica Scandinavica Supplement 230, 1-54.

Cholewicki, J., McGill, S.M., 1996. Mechanical stability of the in vivo lumbar spine: implications for injury and chronic low back pain. Clinical Biomechanics 11, 1-15.

Crisco 3d, J.J., Panjabi, M.M., 1991. The intersegmental and multisegmental muscles of the lumbar spine. A biomechanical model comparing lateral stabilizing potential. Spine 16 (7), 793-799.

Crisco, J.J., Panjabi, M.M., Yamamoto, I., Oxland, T.R., 1992. Euler stability of the human ligamentous lumbar spine. Part 2: experimental. Clinical Biomechanics 7, 27-32.

Crowninshield, R.D., Brand, R.A., 1981. A physiologically based criterion of muscle force prediction in locomotion. Journal of Biomechanics 14, 793-801. van Dieën, J.H., 1997. Are recruitment patterns of the trunk musculature compatible with a synergy based on the maximization of endurance? Journal of Biomechanics 30, 1095-1100.

Dul, J., Johnson, G.E., Shiavi, R., Townsend, M.A., 1984. Muscular synergism. II. A minimum fatigue criterion for load sharing between synergistic muscles. Journal of Biomechanics 17, 675-684.

Gardner-Morse, M., Stokes, I.A.F., Laible, J.P., 1995. Role of muscles in lumbar spine stability in maximum extension efforts. Journal of Orthopaedic Research 13 (5), 802-808.

Gardner-Morse, M.G., Stokes, I.A.F., 1998. The effects of abdominal muscle coactivation on lumbar spine stability. Spine 23 (1), 86-92.

Hughes, R.E., Chaffin, D.B., Lavender, S.A., Andersson, G.B., 1994. Evaluation of muscle force prediction models of the lumbar trunk using surface electromyography. Journal of Orthopaedic Research. $12(5), 689-698$.

Inman, D.J., 1989. Vibration with Control, Measurement, and Stability. Prentice Hall, Englewood Cliffs, NJ, USA, pp. 70-72.

Lavender, S.A., Tsuang, Y.H., Andersson, G.B., Hafezi, A., Shin, C.C., 1992. Trunk muscle coactivation: the effects of moment direction and moment magnitude. Journal of Orthopaedic Research 10, 691-700.

Panjabi, M.M., 1992. The stabilizing system of the spine. Part II. Neutral zone and instability hypothesis. Journal of Spinal Disordorders 5 (4), 390-396.

Schultz, A.B., 1990. Biomechanical analyses of loads on the lumbar spine. In: Weinstein, J.N., Wiesel, S.W. (Eds.), The Lumbar Spine. WB Saunders, Philadelphia, pp. 160-171.

Skogland, L.B., Miller, J.A.A., 1980. On the importance of growth in idiopathic scoliosis: a biochemical, radiological and biomechanical study. Ph.D. Dissertation, University of Oslo, Oslo, Norway.

Stokes, I.A.F., Gardner-Morse, M., 1995. Lumbar spine maximum efforts and muscle recruitment patterns predicted by a model with multijoint muscles and flexible joints. J Biomechanics 28 (2), 173186.

Stokes, I.A.F., Gardner-Morse, M., 1999. Quantitative anatomy of the lumbar musculature. Journal of Biomechanics 32, 311-316.

Stokes, I.A.F., Gardner-Morse, M., Henry, S., Badger, G.J., 2000. Trunk muscular response to perturbation with preactivation of lumbar spinal musculature. Spine 25 (15), 1957-1964.

Thelen, D.G., Schultz, A.B., Ashton-Miller, J.A., 1995. Co-contraction of lumbar muscles during the development of time-varying triaxial moments. Journal of Orthopaedic Research 13 (3), 390-398.

Wynarsky, G.T., Schultz, A.B., 1991. Optimization of skeletal configuration: studies of scoliosis correction biomechanics. Journal of Biomechanics 24 (8), 721-732.

Yettram, A.L., Jackman, M.J., 1982. Structural analysis for the forces in the human spinal column and its musculature. Journal of Biomedical Engineering 4 (2), 118-124. 